\title{
Dietary Patterns and Risk of Micronutrient Deficiencies: their Implication for Nutritional Intervention in Ethiopia
}

\author{
Tibebeselassie Seyoum Keflie ${ }^{1^{*}}$, Aregash Samuel${ }^{2}$, Christine Lambert ${ }^{3}$, \\ Donatus Nohr ${ }^{4}$ and Hans Konrad Biesalski ${ }^{5}$ \\ ${ }^{1}$ Ph.D. Candidate, Institute of Biological Chemistry and Nutrition (140a), University of Hohenheim, Stuttgart, Germany \\ ${ }^{2}$ Researcher, Ph.D. Candidate, Ethiopian Public Health Institute, Gulelle Arbegnoch Street, Gulelle Subcity, Addis Abeba, Ethiopia \\ ${ }^{3}$ Scientific staff, Ph.D. Institute of Biological Chemistry and Nutrition (140a), University of Hohenheim, Stuttgart, Germany \\ ${ }^{4}$ Professor, Scientific staff, Institute of Biological Chemistry and Nutrition (140a), University of Hohenheim, Stuttgart, Germany \\ ${ }^{5}$ Professor of nutritional medicine, former director, Institute of Biological Chemistry and Nutrition (140a), University of \\ Hohenheim, Stuttgart, Germany
}

Received: December 22, 2017; Accepted: January 15, 2018; Published: January 31, 2018

*Corresponding author: Tibebeselassie Seyoum Keflie, Ph.D. Candidate, Postal Address: Garbenstr. 30, 70593 Stuttgart, Germany, Tel: +4917658995469; Fax:+4971145924830, E-mail: tibebe.fscuhoh@gmail.com, Tibebeselassie_Keflie@uni-hohenheim.de

\section{Abstract}

Background: Dietary patterns are the quantities, proportions, variety or combinations of different foods and beverages in diets, and the frequency with which they are habitually consumed. In Ethiopia, information on the dietary patterns and association of the proxies of dietary adequacy and quality with nutritional outcomes is scarce. The aims of this paper were to assess the interactions between dietary patterns, nutritional adequacy, nutritional quality and the risk of micronutrient deficiencies, and to highlight their implications in nutritional interventions.
\end{abstract}

Methods: A community based cross-sectional study was carried out in North Shewa zone of Amhara Regional State, central Ethiopia from December 2014 to February 2015. Multistage sampling techniques were employed to recruit participants and 640 subjects involved in the study Data were collected using structured and seven-day recall questionnaires. Chi-Square test, Kruskal-Walis test, Spearman correlation, multiple linear and multinomial regression models were used for inferential analyses.

Results: The main dietary patterns included cereals, vegetables and legumes. Animal Source Foods (ASF) was consumed by $35.4 \%$ of participants. The median (range) of Food Variety Score (FVS) and Diet Diversity Score (DDS) were 16 (8-25) and 3.43 (1.14-5.57), respectively. About $28 \%$ of subjects were malnourished. FVS had a positive correlation with DDS ( $\mathrm{r}=0.502, p<0.001)$ and Body Mass Index (BMI) ( $\mathrm{r}=0.145, p<0.001)$. DDS had also a positive correlation with BMI ( $\mathrm{r}=0.19, p<0.001)$. Family size and educational status were identified as determinant factors for FVS, but the later had a significant influence on DDS. The risks of vitamin A and iron deficiencies were $60.3 \%$ and $86.3 \%$, respectively. The consumption of food groups rich in vitamin A and haem iron were significantly different across FVS and DDS $(p<0.05)$.

Conclusions: Dietary inadequacy, poor nutritional quality and high risk of micronutrient deficiencies were identified. These underlined the implications of nutritional interventions in central Ethiopia.

Keywords: dietary patterns; micronutrients; FVS; DDS; BMI; Ethiopia

Abbreviations: ASF - Animal Source Foods; BMI - Body Mass Index; DDS - Diet Diversity Score; FVS - Food Variety Score; LL - Lower Limit; OR - Odds Ratio; UL - Upper Limit; UNICEF - United Nations Children's Fund; WHO - World Health Organization.

\section{Background}

Dietary patterns are the quantities, proportions, variety or combinations of different foods and beverages in diets, and the frequency with which they are habitually consumed. The dietary patterns' approach considers the inherent interactions between foods and nutrients in promoting either health or increasing disease risk [1]. Until quite recently, there has been extensive focus on the quantity of food produced and consumed in food security rhetoric and in policy and decision-making arenas and much less attention given to the nutritional quality of foods and diets [2].
Nutritional deficiencies are not only the result of inadequate food consumed, but also of poor dietary quality and diversity despite adequate calories in many cases [3]. The prevalence of diseases associated with a poor-quality diet is increasing in Ethiopia. Even though most people consume plant based foods, diets low in fruits and vegetables are found to be the most common risk factors contributing to a large portion of dietrelated Non-Communicable Diseases (NCD) [4, 5, 6, 7]. In 2013, more than a third (35.1\%) of all deaths in Ethiopia was caused by NCDs [7]. The emergence of NCDs imposes another burden on the country's health system while it is still striving to address infectious diseases and under nutrition. 
Understanding the dietary patterns and evaluating their qualities are essential for nutritional intervention. The quality of diet can be assessed using a simple score of foods variety and dietary diversity [8]. Food variety is expressed as the number of biologically distinct foods eaten over a designated period. It minimizes the adverse consequences of food on health; and reduces the risk of NCDs [9]. It is usually quantified by the number of food items compared with the number of nutritious food groups known as dietary diversity $[10,11]$.

Assessing Food Variety Score (FVS) is a quick, simple and low-cost method of determining the nutritional adequacy of a diet. It is believed that a nutritionally adequate diet is best achieved by consuming a diverse range of foods [12]. Likewise, individual Diet Diversity Score (DDS) is a simple proxy measure of the nutritional quality of individual's diets, particularly that of micronutrient adequacy of a diet [13]. Both FVS and DDS reflect the quality of the diet. Scores of dietary diversities have been positively correlated with macro and micronutrient adequacy of the adolescents and adults $[9,14,15]$. Savy et, al. described the importance of studying the association between proxies of overall dietary quality and nutritional outcomes [8]. Workicho et, al. also highlighted the need of tracking dietary quality and progress in nutritional outcomes in a population to develop timely interventions [16].

Until recent time, very few studies have been conducted in Ethiopia in relation to balanced and diversified diets. Of these studies, none has attempted to point out the implication of dietary patterns and risks of micronutrient deficiencies on nutritional intervention. Therefore, the aims of the present study were:

- to assess the dietary patterns, nutritional adequacy and nutritional quality of the populations;

- to examine their relationship with nutritional status; and

- to describe the implications of the outcomes in nutritional interventions in Ethiopia.

\section{Materials and Methods}

\section{Study Area and Subjects}

This study was conducted as a community based crosssectional study in North Shewa zone of Amhara Regional State, Central Ethiopia from December 2014 to February 2015. Based on the 2007 census, 928,694 men and 908,796 women with a total of $1,837,490$ people inhabited in this area [17]. Multistage sampling techniques were used to recruit study subjects. First, all kebeles, which are the smallest administrative unit, were stratified into urban and rural settings. Second, four kebeles from urban and three kebeles from rural settings were selected by simple random sampling technique. Third, households were included from each kebele by systematic random sampling technique. The first household was selected by a lottery system and then, every third household was included in the study. Lastly, one study subject was randomly selected from each household.

The criteria to include study subjects were age above 18 years, living in the house for at least 6 months and willing to participate in the study. Subjects who were absent during the survey, disabled, seriously ill or had some difficulty of communication were excluded. Single population proportion formula was used to determine the sample size. Taking the assumptions of $50 \%$ dietary intake below average DDS with 95\% confidence interval, $4 \%$ margin of error and $10 \%$ drop out rate, a sample size of 660 was obtained. However, we recruited 100 study subjects from each kebele with a total of 700 study subjects to participate in the study.

\section{Data collection}

The data were collected by seven health extension workers who have been graduated with diploma (2 to 3 years' college training) in nursing. Questionnaires which comprised of different parts were translated into Amharic, the local language. In the structured questionnaire, there were socio-demographic and anthropometric parts, whereas; in the dietary diversity questionnaire, the dietary intake assessment part was included. All interviewers were given one-day training on the content of the questionnaires and on the techniques, how to ask the list of ingredients for composite dishes and how to probe for meals and snacks not indicated in the list of 7-day recall. Before instigating the interview, each of them practiced on the questionnaire to alleviate ambiguity and minimize errors as much as possible.

\section{Dietary Assessment}

Seven-day recall of dietary intake was carried out on the study subjects. The dietary diversity questionnaire, which was adapted from the guidelines for measuring household and individual dietary diversity, was employed for face-to-face interview [12]. Each subject was asked to describe what he or she ate and/or drank for breakfast, snack, lunch, snack and dinner whether at home or outside the home for the past seven days. The interviews included a detailed description of foods consumed, the ingredients used, the cooking method, and brand names (for packed foods). The food items were subsequently transformed into food groups and their frequencies of consumption were computed and used for further analyses Table 1.

\section{Food Variety Score (FVS)}

FVS was measured using simple count of individual food items consumed during the seven days. Food variety checklist developed by Savige et, al. was used to score the food items [12]. Each type of food consumed is scored once over a week time. The maximum score would be 57. Quantities smaller than 1-2 tablespoons (except for fats and oils) do not represent a sufficient quantity to rate FVS. The results of FVS were categorized into five dietary adequacy groups: very poor ( $<10 \mathrm{FVS}$ per week), poor (10-19 FVS per week), fair (20-24 FVS per week), good (25-30 FVS per week) and very good (>30 FVS per week) [12].

\section{Diet diversity score}

Individual DDS was calculated as the number of food groups consumed during the first day recall. These food groups were 
Dietary Patterns and Risk of Micronutrient Deficiencies: their Implication for Nutritional Intervention in Ethiopia

Table 1: Food groups used for dietary diversity score*

\begin{tabular}{|c|c|c|c|}
\hline Number & Food groups & Subgroups & $\begin{array}{l}\text { Scores (if consumption is: } \\
\text { yes }=1 \text {, otherwise: } n o=0 \text { ) }\end{array}$ \\
\hline 1 & Starchy staples & $\begin{array}{l}\text { Cereals, grains, white roots and } \\
\text { tubers }\end{array}$ & 1 or 0 \\
\hline 2 & Dark green vegetables & $\begin{array}{l}\text { Locally available vitamin A rich } \\
\text { vegetables such as kale, lettuce, } \\
\text { spinach and wild forms such as } \\
\text { samma (stinging nettle) }\end{array}$ & 1 or 0 \\
\hline 3 & $\begin{array}{c}\text { Other vitamin A rich fruits and } \\
\text { vegetables }\end{array}$ & $\begin{array}{c}\text { vitamin A rich fruits (mango), } \\
\text { vegetables (carrot) and tubers } \\
\text { (vitamin A blended sweet potatoes) }\end{array}$ & 1 or 0 \\
\hline \multirow{2}{*}{4} & \multirow{2}{*}{ Other fruits and vegetables } & $\begin{array}{c}\text { Fruits: such as avocados, banana, } \\
\text { dates, etc. }\end{array}$ & \multirow{2}{*}{1 or 0} \\
\hline & & $\begin{array}{l}\text { Vegetables: such as cabbage, onion, } \\
\text { garlic, green pepper, tomatoes, etc. }\end{array}$ & \\
\hline 5 & Organ meat & $\begin{array}{l}\text { Red organ meats such as liver, kidney, } \\
\text { heart and any processed organ meats }\end{array}$ & 1 or 0 \\
\hline 6 & Meat and fish & $\begin{array}{l}\text { Beef, lamb, goat meat, chicken and } \\
\text { fresh fish }\end{array}$ & 1 or 0 \\
\hline 7 & Eggs & Chicken eggs, quail eggs & 1 or 0 \\
\hline \multirow[t]{2}{*}{8} & \multirow[t]{2}{*}{ Legumes, nuts and seeds } & $\begin{array}{l}\text { Legumes/pulses: such as beans, peas, } \\
\text { lentils, peanuts, etc. }\end{array}$ & \multirow[t]{2}{*}{1 or 0} \\
\hline & & $\begin{array}{l}\text { Seeds: such as oil seeds and pumpkin } \\
\text { seeds }\end{array}$ & \\
\hline 9 & Milk and milk products & $\begin{array}{l}\text { Dairy products such as milk, butter, } \\
\text { sour milk, butter milk, cheese and } \\
\text { whey }\end{array}$ & 1 or 0 \\
\hline
\end{tabular}

based on the guidelines for measuring household and individual dietary diversity Table 1 . The score for individual diet diversity goes from 0 to 9 [13]. The percentages of the consumption of food groups rich in micronutrients such as vitamin A or iron were calculated using the food groups of DDS

\section{Anthropometric Measurements}

The weights of the subjects were measured while they were dressed in light clothes to the nearest of $100 \mathrm{~g}$. SECA personal weighing scale (used by UNICEF) was employed for measurement. The heights of the subjects were measured using tape meter fixed on the wall without shoes to the nearest of 0.1 $\mathrm{cm}$. For the calculation of Body Mass Index (BMI), the weight of the subject (in $\mathrm{kg}$ ) was divided by the height (in meter) squared of the subject. BMI was described as underweight $(<18.50 \mathrm{~kg} /$ $\left.\mathrm{m}^{2}\right)$, normal weight $\left(18.50-24.99 \mathrm{~kg} / \mathrm{m}^{2}\right)$, overweight $(25-30 \mathrm{~kg} /$ $\left.\mathrm{m}^{2}\right)$ and obese $\left(>30 \mathrm{~kg} / \mathrm{m}^{2}\right)[18]$.

\section{Statistical Analysis}

Statistical analysis was carried out using IBM SPSS version 23 statistical program. Continuous data were checked for normality using Kolmogorov-Smirnov test. When data were not normally distributed ( $\mathrm{p}>0.05)$, nonparametric tests were used. In descriptive summaries, median (range) and percentages were used to present the data. Inferential statistics were also employed. The difference between proportions of categorical variables was examined by Chi-Square test. Kruskal-Wallis test was used to determine the differences between socio demographic variables on FVS, DDS and BMI.

Bivariate analyses were carried out to test the links between socio demographic variables and dietary scores. The socio demographic variables which were significantly linked to either dietary scores or BMI were selected as potential confounders $(P<0.15)$. Significant variables subsequently included into the 
multivariate analysis in order to better identify the collinearities between variables $(\mathrm{P}<0.05)$. Multiple linear regression model was employed to differentiate the independent predictors of FVS and DDS after adjusting for confounding factors.

Spearman correlation was used to examine the association between FVS, DDS, BMI, age and average meal frequency. The relationships between the groups of FVS, DDS and BMI were analysed using a multinomial logistic regression model. Odds Ratio (OR) was used to report the strength of association between the proportions of vitamin $\mathrm{A}$ and haem iron rich foods consumption between urban and rural areas. Unless specified, $p$ value $<0.05$ was considered as statistically significant.

\section{Results}

\section{Socio-demographic study}

The study was undertaken in seven kebeles in urban and rural areas of North Shewa zone of Amhara Regional State, Central Ethiopia. A total of 700 participants were recruited. But, 640 were involved in the study with a response rate of 91.4\%. The remaining $8.6 \%$ were excluded because of their refusal and absence during the study period. Table 2 presents the socio-demographic characteristics of the study participants. Most participants belonged to Amhara ethnic group (93.5\%) and Orthodox Tewahido Christian (89.8\%). The median age

Table 2: Socio demographic characteristics

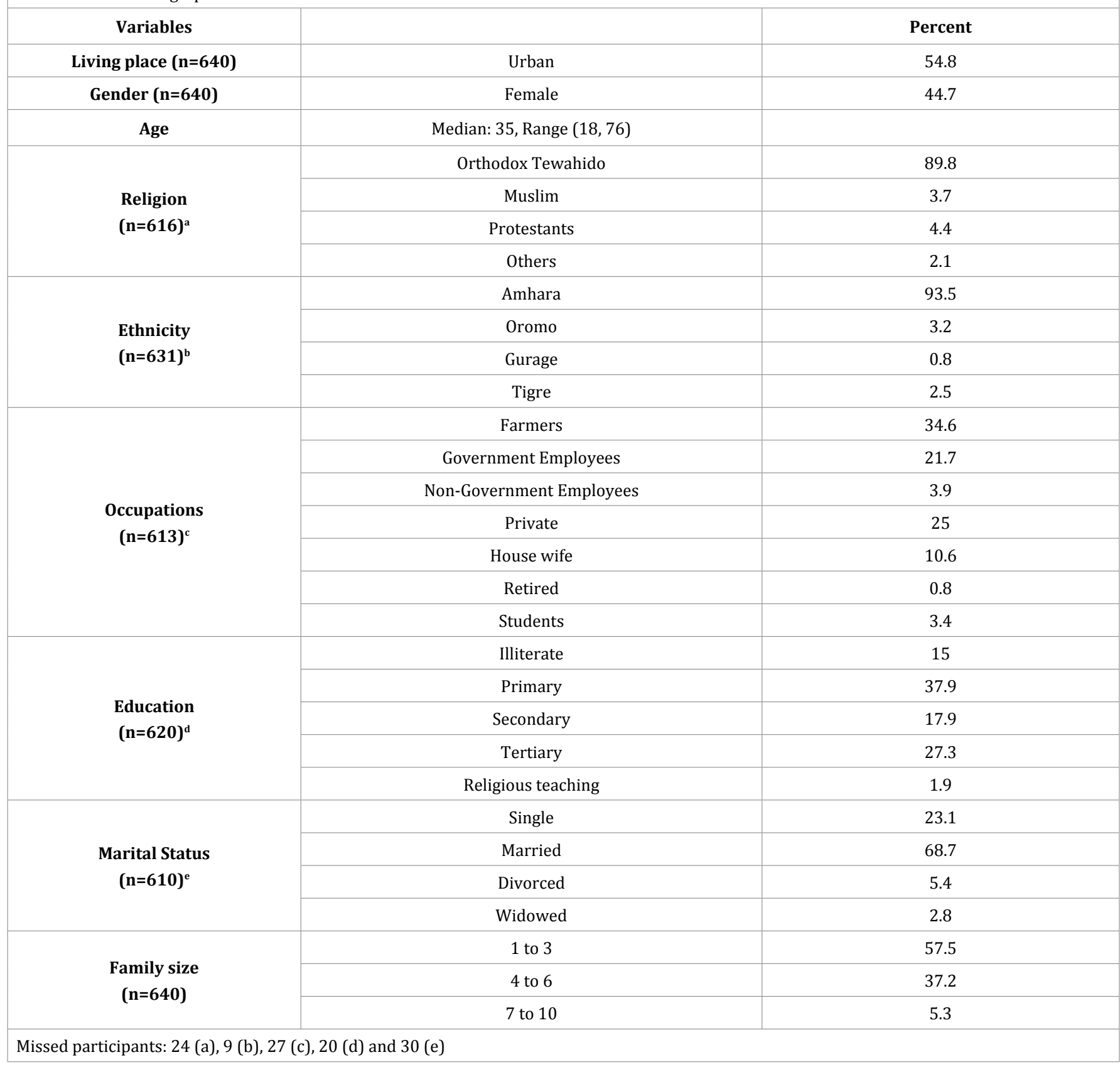


was 35 years (range= 18 to 76 years). A little below half of the participants were females (44.7\%), 54.8\% were urban dwellers, $34.6 \%$ were farmers, $62.1 \%$ had at least primary education, $68.7 \%$ were married and $57.5 \%$ had 1 to 3 family size.

\section{Dietary patterns}

About 130 food items were identified in the study areas (See additional file 1). The main dietary patterns were included cereals, vegetables and legumes. Almost all subjects consumed starchy staples $(99.7 \%)$ and about 58\% consumed legumes cooked with oils and fats (99.2\%) for the whole week Table 3. In every day meal, $95.6 \%$ consumed the food group of other fruits and vegetables Table 4. Dairy products (62.4\%), dark green vegetables (49.69\%), meat (37.9\%) and, other vitamin A rich fruits and vegetables (30.35\%) were consumed at least once in a week Table 4,5 . On the other hand, organ meat $(2.6 \%)$ and fish $(3.6 \%)$ were seldom consumed by the subjects. The food consumption patterns were significantly different between urban and rural areas $(P<0.05)$.

Additional file 1: Food items identified in North Shewa, Ethiopia

\begin{tabular}{|c|c|c|}
\hline Cereals and grains & & Legumes/pulses \\
\hline Ambasha, circular flat bread dough & Kinche, boiled splitted barley served with butter & Ashuk, roasted and boiled faba bean \\
\hline $\begin{array}{l}\text { Anebabero, double injera covered } \\
\text { with butter and chilli in the middle }\end{array}$ & Kinche, boiled splitted wheat served with butter & Bokolt, germinated faba bean \\
\hline Atmit, very thin barley porridge & Kita, unleavened flat barley bread & Endushdush, soaked and roasted faba bean \\
\hline Atmit, very thin wheat porridge & Kita, unleavened flat teff bread & Fool, pureed stewed faba bean \\
\hline $\begin{array}{c}\text { Besso, roasted and milled } \\
\text { barleyflour served with butter }\end{array}$ & Kita, unleavened flat wheat bread & Nifro, boiled chickpea \\
\hline $\begin{array}{l}\text { Biscuit, homemade fried dough } \\
\text { bread }\end{array}$ & Kolo, roasted and mixed barley, chickpea and pea & Nifro, boiled faba bean \\
\hline $\begin{array}{l}\text { Bonbolino, homemade fried dough } \\
\text { bread containing sugar }\end{array}$ & Kolo, roasted and mixed wheat, chickpea and sunflower & Nifro, boiled faba bean and maize \\
\hline Bread, wheat & Kolo, roasted barley & Nifro, boiled faba bean and wheat \\
\hline Cake & Kolo, roasted chickpea & Shorba, lentil, carrot, and macaroni soup \\
\hline $\begin{array}{l}\text { Chechebisa, pieces of barley bread } \\
\text { mixed with butter }\end{array}$ & Kolo, roasted pea & Shorba, lentil, pea and carrot soup \\
\hline $\begin{array}{l}\text { Chechebisa, pieces of wheat bread } \\
\text { mixed with butter }\end{array}$ & Kolo, roasted wheat & $\begin{array}{l}\text { Siljo, fermented faba bean, sunflower and } \\
\text { mustard slurry }\end{array}$ \\
\hline Cukis & Macaroni & Wot, beyeayinet -varieties of stews \\
\hline $\begin{array}{l}\text { Dabo-kolo, very small size roasted } \\
\text { bread dough }\end{array}$ & Nifro, boiled wheat & Wot, faba bean, chili, onion and oil stew \\
\hline $\begin{array}{l}\text { Fetira, fried filo dough cooked with } \\
\text { egg and covered with honey }\end{array}$ & Pizza & Wot, pea flour, onion, chili and oil stew \\
\hline $\begin{array}{l}\text { Firfir, pieces of barley injera with } \\
\text { stew }\end{array}$ & Porridge, barley served with butter and chili & Wot, splitted lentil, chili, onion and oil stew \\
\hline $\begin{array}{l}\text { Firfir, pieces of bread with stew } \\
\text { containing butter }\end{array}$ & Porridge, wheat served with butter and chili & Wot, splitted pea, onion, chili and oil stew \\
\hline $\begin{array}{l}\text { Firfir, pieces of teff injera with stew } \\
\text { containing beef }\end{array}$ & Sambusa & Wot, splitted pea, onion, oil and turmeric stew \\
\hline $\begin{array}{l}\text { Firfir, pieces of teff injera with stew } \\
\text { containing butter }\end{array}$ & Sandwich, sliced bread with fried egg in the middle & $\begin{array}{l}\text { Wot, tegabino - pea flour, onion, chili, garlic } \\
\text { and oil stew }\end{array}$ \\
\hline $\begin{array}{l}\text { Firfir, pieces of teff injera with stew } \\
\text { containing dried beef }\end{array}$ & Spaghetti, pasta & Wot, whole lentil, onion and oil stew \\
\hline $\begin{array}{l}\text { Fitfit, pieces of teff injera mixed with } \\
\text { beef broth }\end{array}$ & Steamed rice & \\
\hline $\begin{array}{l}\text { Fitfit, pieces of teff injera mixed with } \\
\text { pea flour, onion and oil sauce }\end{array}$ & Tirosho, flat barley bread dough covered with butter & \\
\hline
\end{tabular}




\begin{tabular}{|c|c|c|}
\hline $\begin{array}{l}\text { Fitfit, pieces of teff injera mixed with } \\
\text { sunflower sauce }\end{array}$ & Tirosho, flat wheat bread dough covered with butter & \\
\hline \multicolumn{3}{|l|}{ Injera, barley } \\
\hline \multicolumn{3}{|l|}{ Injera, teff } \\
\hline \multicolumn{3}{|l|}{ Injera, wild oat } \\
\hline Vegetables and tubers & Meat & Sugar / Confectionary \\
\hline Atkilt, mixed vegetables and fruits & Beef with steamed rice & Sugar \\
\hline $\begin{array}{l}\text { Bula - false banana porridge served } \\
\text { with butter }\end{array}$ & $\begin{array}{l}\text { Dulet, semi roasted organ meat (sheep and goat) with } \\
\text { butter }\end{array}$ & Honey \\
\hline Ethiopian kale & Kikil, boiled beef & Sugar cane \\
\hline Fried potatoes & Kikil, boiled egg & Salts and spices \\
\hline Kariya, green pepper & Kikil, boiled goat meet & Salt \\
\hline $\begin{array}{l}\text { Kariya, sinig - green pepper stuffed } \\
\text { with onion and oil }\end{array}$ & Kikil, boiled mutton & Bird's eye chili \\
\hline Kikil, boiled potatoes & $\begin{array}{l}\text { Kitfo, raw or sautéed minced beef mixed with chili and } \\
\text { clarified spicy butter }\end{array}$ & Bishop's weed \\
\hline Kikil, boiled sugar beet & Milas na senber, roasted cow tongue and rumen & Black cumin \\
\hline $\begin{array}{l}\text { Lettuce with onion, oil and aceto } \\
\text { vinegar }\end{array}$ & Raw beef & Cardamom \\
\hline $\begin{array}{l}\text { Raw tomatoes with onion, green } \\
\text { peppers and oil }\end{array}$ & Roasted beef & Cinnamon \\
\hline Samma, Stinging nettle & Roasted goat meat & Cloves \\
\hline Shorba, vegetables soup & Roasted mutton & Coriander seeds \\
\hline $\begin{array}{l}\text { Sils, roasted tomatoes with onion, } \\
\text { oil and green pepper }\end{array}$ & Shoriba, beef broth & Rue \\
\hline Swiss chard & Wot, beef with kale & Alcohol beverages \\
\hline Wot, beetroot, onion and oil stew & Wot, minced beef and egg stew & Tela, local beer \\
\hline Wot, cabbage, onion and oil stew & Wot, red beef stew & Keribo, hops free local drinks \\
\hline $\begin{array}{l}\text { Wot, cabbage, potatoes, carrot, } \\
\text { onion and oil stew }\end{array}$ & Wot, red chicken stew & Tej, mead honey or sugary wine \\
\hline Wot, carrot, onion and oil stew & Wot, red dried beef stew & Areke, homemade hard liquour \\
\hline Wot, kale, garlic, onion, and oil stew & Wot, red mutton stew & Wine \\
\hline $\begin{array}{l}\text { Wot, potatoes, onion, chili and oil } \\
\text { stew }\end{array}$ & Eggs & Beer \\
\hline $\begin{array}{l}\text { Wot, pumpkin, chili, onion and oil } \\
\text { stew }\end{array}$ & Chiken eggs & \\
\hline $\begin{array}{l}\text { Wot, stinging nettle and barley flour } \\
\text { stew }\end{array}$ & Dairy products & \\
\hline Wot, Swiss chard, onion and oil stew & Aguat, whey & \\
\hline $\begin{array}{l}\text { Wot, tomatoes, chili, onion and oil } \\
\text { stew }\end{array}$ & Arera, butter milk & \\
\hline Fruits & Ayib, Cheese & \\
\hline Avocado & Butter & \\
\hline
\end{tabular}


Dietary Patterns and Risk of Micronutrient Deficiencies: their Implication for Nutritional Intervention in Ethiopia

\begin{tabular}{|c|c|c|}
\hline Banana & Milk & Yoghurt \\
\hline Juice, mixed & Fish & \\
\hline Juice, avocado & Fresh fish & \\
\hline Juice, mango & Fats and oil & Oil \\
\hline Mango & Butter & \\
\hline Orange & \\
\hline Temir, dates & \\
\hline
\end{tabular}

Table 3: Percentages of the consumption frequencies of starchy staples, legumes and others per week

\begin{tabular}{|c|c|c|c|c|c|}
\hline \multicolumn{2}{|c|}{ Food groups } & Urban $(n=347)$ & Rural (n=289) & Total $(n=636)$ & P-value \\
\hline \multirow{2}{*}{ Starchy staples } & 1-3 days & 0 & 0.7 & 0.3 & 0.206 \\
\hline & The whole week & 100 & 99.3 & 99.7 & \\
\hline \multirow{4}{*}{ Legumes, nuts and seeds } & None & 0.6 & 0 & 0.3 & $<0.001^{*}$ \\
\hline & $1-3$ days & 6.1 & 1.4 & 3.9 & \\
\hline & 4-6 days & 50.1 & 23.2 & 37.9 & \\
\hline & The whole week & 43.2 & 75.4 & 57.9 & \\
\hline \multirow{3}{*}{ Oils and fats } & None & 0.9 & 0 & 0.5 & 0.086 \\
\hline & 1-3 days & 0 & 0.7 & 0.3 & \\
\hline & The whole week & 99.1 & 99.3 & 99.2 & \\
\hline \multirow{4}{*}{$\begin{array}{l}\text { Spices, condiments and } \\
\text { beverages }\end{array}$} & None & 0.3 & 0 & 0.2 & 0.285 \\
\hline & 1-3days & 0 & 0.7 & 0.3 & \\
\hline & 4-6days & 0.3 & 0.7 & 0.5 & \\
\hline & The whole week & 99.4 & 98.6 & 99.1 & \\
\hline
\end{tabular}

Table 4: Percentages of the consumption frequencies of fruits and vegetables per week

\begin{tabular}{|c|c|c|c|c|c|}
\hline Food groups & & Urban $(n=347)$ & Rural (n=289) & Total $(n=636)$ & P-value \\
\hline \multirow{4}{*}{ Dark green vegetables } & None & 46.4 & 57.1 & 51.3 & $0.001^{*}$ \\
\hline & 1-3 days & 42.9 & 39.4 & 41.4 & \\
\hline & 4-6 days & 8.6 & 3.5 & 6.3 & \\
\hline & The whole week & 2 & 0 & 1.1 & \\
\hline \multirow{4}{*}{$\begin{array}{l}\text { Other vitamin A rich fruits } \\
\text { and vegetables }\end{array}$} & None & 58.5 & 83 & 69.7 & $<0.001^{*}$ \\
\hline & 1-3 days & 35.2 & 15.9 & 26.4 & \\
\hline & 4-6 days & 4.6 & 0.7 & 2.8 & \\
\hline & The whole week & 1.7 & 0.3 & 1.1 & \\
\hline \multirow{4}{*}{ Vegetables } & None & 0.6 & 0 & 0.3 & $<0.001^{*}$ \\
\hline & 1-3 days & 0.6 & 0.7 & 0.6 & \\
\hline & 4-6 days & 3.4 & 26 & 13.6 & \\
\hline & The whole week & 95.4 & 73.4 & 85.5 & \\
\hline
\end{tabular}


Dietary Patterns and Risk of Micronutrient Deficiencies: their Implication for Nutritional Intervention in Ethiopia

\begin{tabular}{|c|c|c|c|c|c|}
\hline \multirow{3}{*}{ Fruits } & None & 85.1 & 97.2 & 90.6 & $<0.001^{*}$ \\
\cline { 2 - 6 } & $1-3$ days & 13.4 & 2.8 & 8.6 & 0.5 \\
\cline { 2 - 6 } & $4-6$ days & 0.9 & 0 & 0.3 & \\
\cline { 2 - 7 } & The whole week & 0.6 & 0 & \\
\hline *Statistically significant
\end{tabular}

Table 5: Percentages of the consumption frequencies of meat, organ meat, eggs and dairy per week

\begin{tabular}{|c|c|c|c|c|c|}
\hline \multicolumn{2}{|c|}{ Food groups } & \multirow{2}{*}{$\begin{array}{c}\text { Urban }(\mathbf{n}=\mathbf{3 4 7}) \\
47.6\end{array}$} & \multirow{2}{*}{$\begin{array}{c}\text { Rural (n=289) } \\
79.6\end{array}$} & \multirow{2}{*}{$\begin{array}{c}\text { Total }(\mathbf{n}=\mathbf{6 3 6}) \\
62.1\end{array}$} & \multirow{2}{*}{$\begin{array}{l}\text { P-value } \\
<0.001^{*}\end{array}$} \\
\hline \multirow{4}{*}{ Meat } & None & & & & \\
\hline & 1-3 days & 40.3 & 19.4 & 30.8 & \\
\hline & 4-6 days & 11 & 1 & 6.5 & \\
\hline & The whole week & 1.2 & 0 & 0.6 & \\
\hline \multirow{4}{*}{ Organ meat } & None & 95.7 & 99.7 & 97.5 & $0.003^{*}$ \\
\hline & 1-3 days & 4 & 0 & 2.2 & \\
\hline & 4-6 days & 0.3 & 0 & 0.2 & \\
\hline & The whole week & 0 & 0.3 & 0.2 & \\
\hline \multirow{3}{*}{ Eggs } & None & 73.2 & 93.8 & 82.5 & $<0.001^{*}$ \\
\hline & 1-3 days & 25.9 & 6.2 & 11 & \\
\hline & 4-6 days & 0.9 & 0 & 0.5 & \\
\hline \multirow{5}{*}{ Dairy products } & None & 44.4 & 29.4 & 37.6 & $<0.001^{*}$ \\
\hline & 1-3 days & 34.3 & 43.6 & 38.5 & \\
\hline & 4-6 days & 19 & 26 & 22.2 & \\
\hline & The whole week & 2.3 & 1 & 1.7 & \\
\hline & & & & & \\
\hline
\end{tabular}

\section{Meal Frequency}

The median meal frequency was 3 (range $=1$ to 5 ). Comparing its distribution, average meal frequency had statistically significant difference across urban and rural areas $(P<0.001)$.

\section{Food variety score}

The median FVS was 16 (range= 8 to 25 ) per week. Urban and rural dwellers had a significant difference in FVS $(P<0.001)$ Table 6. More than $98 \%$ of participants had poor and fair FVS in the areas Figure 1. There was also a significant FVS difference between socio demographic variables such as occupation $(\mathrm{P}<0.001)$, educational status $(\mathrm{P}<0.001)$ and family size $(\mathrm{P}<0.001)$. Spearman's rank correlation showed that FVS had a positive association with DDS $(\mathrm{r}=0.502, \mathrm{P}<0.001)$ and $\mathrm{BMI}(\mathrm{r}=$ $0.145, \mathrm{p}<0.001$ ). However, it had a negative correlation with average meal frequency $(\mathrm{r}=-0.102, \mathrm{P}=0.01)$. The results of regression model revealed that educational status and family size had significant influences on FVS $(\mathrm{P}<0.05)$ Table 7.

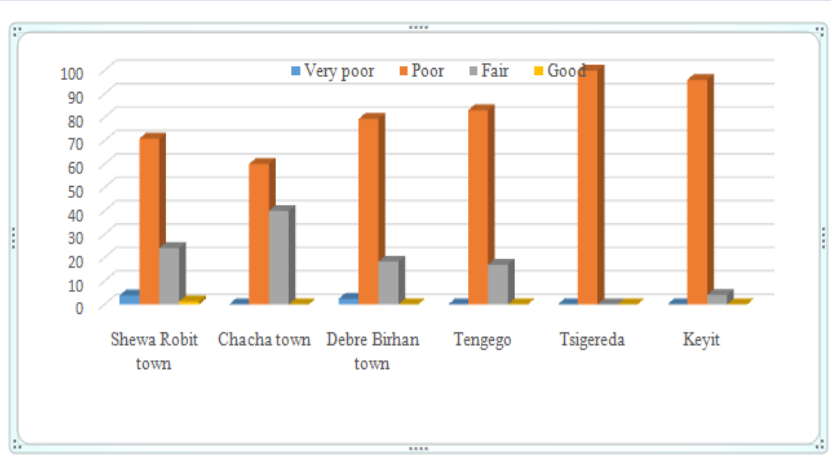

Figure 1: Food variety scores in different locations

\section{Diet diversity score}

The median DDS was 3.43 score (range $=1.14$ to 5.57 ). The DDS of urban and rural dwellers was significantly different $(P=0.004)$ Table 6 . Nearly, $41 \%$ of subjects had $2.50-3.50$ scores 
Table 6: FVS, DDS and BMI distribution across urban and rural settings

\begin{tabular}{|c|c|c|c|c|c|c|c|}
\hline Variables & \multicolumn{3}{|c|}{ Urban } & \multicolumn{3}{c|}{ Rural } & P-value \\
\hline & $\mathrm{N}$ & Mean & $\mathrm{SD}$ & $\mathrm{N}$ & Mean & SD & \\
\hline FVS & 348 & 17.09 & 3.35 & 289 & 15.31 & 2.51 & $<0.001^{*}$ \\
\hline DDS & 348 & 3.63 & 0.63 & 289 & 3.45 & 0.38 & $0.004^{*}$ \\
\hline BMI & 340 & 22.96 & 3.86 & 250 & 22.31 & 3.2 & $0.028^{*}$ \\
\hline *Statistically significant & & & & &
\end{tabular}

Table 7: Prediction of the effect of explanatory variables on dietary scores and BMI using multiple linear regression model

\begin{tabular}{|c|c|c|c|c|c|c|}
\hline \multirow{2}{*}{ Explanatory variables } & FVS & & DDS & & BMI & P-value \\
\cline { 2 - 7 } & $\beta$ (standardized) & P-value & $\beta$ (standardized) & P-value & $\beta$ (standardized) & 0.082 \\
\hline Occupations & 0.046 & 0.247 & 0.01 & 0.811 & 0.075 & 0.230 \\
\hline Educational status & 0.198 & $<0.0001^{*}$ & 0.122 & $0.004^{*}$ & 0.054 & 0.836 \\
\hline Family size & -0.171 & $<0.0001^{*}$ & -0.045 & 0.292 & -0.009 & 0.01 \\
\hline $\mathbf{R}^{2}$ & 0.094 & & 0.021 & & & \\
\hline
\end{tabular}

Table 8: Vitamin A rich food groups used in vitamin A analysis

\begin{tabular}{|c|c|c|c|c|c|}
\hline \multicolumn{3}{|c|}{ Pro-vitamin A } & \multicolumn{3}{|c|}{ Pre-vitamin A } \\
\hline Food group & Food item & $\operatorname{RAE}(\mu \mathrm{g} / \mathbf{1 0 0 g})^{*}$ & Food group & Food item & $\operatorname{RAE}\left(\mu \mathrm{g} / \mathbf{1 0 0 g}^{*}\right.$ \\
\hline \multirow{6}{*}{$\begin{array}{c}\text { Vitamin A rich } \\
\text { vegetables or tubers }\end{array}$} & Carrot, raw & 835 & \multirow{6}{*}{ Organ meat } & Liver (cattle), raw & 4968 \\
\hline & Carrot, cooked & 852 & & Liver (cattle), cooked & 9442 \\
\hline & $\begin{array}{c}\text { Sweet potato (orange or dark } \\
\text { yellow), raw }\end{array}$ & 709 & & Liver (sheep), raw & 7391 \\
\hline & $\begin{array}{l}\text { Sweet potato (orange or dark } \\
\text { yellow), cooked }\end{array}$ & 1043 & & Liver (sheep), cooked & 7491 \\
\hline & Pumpkin, raw & 426 & & Kidney (cattle), raw & 419 \\
\hline & Pumpkin, cooked & 288 & & Kidney (cattle), cooked & 0 \\
\hline \multirow{5}{*}{$\begin{array}{c}\text { Dark green leafy } \\
\text { vegetables }\end{array}$} & Kale, raw & 500 & \multirow{5}{*}{ Eggs } & Kidney (sheep), raw & 95 \\
\hline & Kale, cooked & 681 & & Kidney (sheep), cooked & 137 \\
\hline & Spinach, raw & 469 & & Chicken eggs, raw & 160 \\
\hline & Spinach, cooked & 524 & & $\begin{array}{l}\text { Chicken eggs, cooked, } \\
\text { fried }\end{array}$ & 219 \\
\hline & Lettuce, raw & 370 & & Quail eggs & 156 \\
\hline \multirow{6}{*}{ Vitamin A rich fruits } & Apricots & 96 & \multirow{6}{*}{$\begin{array}{l}\text { Milk and milk } \\
\text { products }\end{array}$} & Milk, low fat & 71 \\
\hline & Mango & 54 & & Butter & 684 \\
\hline & Papaya & 47 & & Sour milk, cultured & 124 \\
\hline & & & & Butter milk, whole & 47 \\
\hline & & & & Cheese, fat free & 11 \\
\hline & & & & Whey, acid fluid & 2 \\
\hline
\end{tabular}


Dietary Patterns and Risk of Micronutrient Deficiencies: their Implication for Nutritional Intervention in Ethiopia

\begin{tabular}{|c|c|c|c|c|c|}
\hline Food groups & Food items & Haem iron $(\mathrm{mg} / \mathbf{1 0})^{*}$ & Food groups & Food items & $\begin{array}{l}\text { Haem iron } \\
(\mathrm{mg} / \mathbf{1 0 0 g})^{*}\end{array}$ \\
\hline \multirow{13}{*}{ Organ meat } & Liver (cattle), raw & 4.9 & \multirow{7}{*}{ Flesh meat } & $\begin{array}{l}\text { Beef (meat and by } \\
\text { products), raw }\end{array}$ & 5.67 \\
\hline & Liver (cattle), cooked & 6.54 & & $\begin{array}{l}\text { Beef, chuck for stew, all } \\
\text { grades, cooked }\end{array}$ & 2.96 \\
\hline & Liver (sheep), raw & 7.37 & & Goat, raw & 2.83 \\
\hline & Liver (sheep), cooked & 8.28 & & Goat roasted & 3.73 \\
\hline & Kidney (cattle), raw & 4.6 & & Lamb, ground, raw & 1.55 \\
\hline & Kidney (cattle), cooked & 5.8 & & Lamb, ground, cooked & 1.79 \\
\hline & Kidney (sheep), raw & 6.38 & & Chicken meat, stewed & 1.17 \\
\hline & Kidney (sheep), cooked & 12.4 & Fish and seafood & Catfish, raw & 0.3 \\
\hline & Heart (cattle), raw & 4.31 & & Catfish, cooked & 1.43 \\
\hline & Heart (cattle), cooked & 6.38 & & Tilapia, raw & 0.56 \\
\hline & Heart (sheep), raw & 4.6 & & $\begin{array}{l}\text { Tilapia, cooked, dry } \\
\text { heated }\end{array}$ & 0.69 \\
\hline & Heart (sheep), cooked & 5.52 & & Mixed species, raw & 0.89 \\
\hline & & & & Mixed species, cooked & 1.14 \\
\hline
\end{tabular}

and $55 \%$ had $3.51-4.50$ scores. Altogether, $96 \%$ of subjects had scores below average DDS (4.5 out of 9 DDS). The bivariate analysis showed that there was statistically significant mean DDS difference between age group $(\mathrm{P}=0.028)$, occupation $(\mathrm{P}=0.027)$, educational status $(<0.001)$, marital status $(\mathrm{P}=0.018)$ and family size (0.004). DDS had also a positive correlation with BMI ( $\mathrm{r}=$ $0.190, P<0.001)$ and average meal frequency $(\mathrm{r}=0.219, \mathrm{P}<0.001)$. After controlling for occupations and family size, educational status had a significant influence on DDS Table 7.

\section{Vitamin A and haem iron indicators}

Table 8 and 9 indicate the food groups that were used for the analyses of the consumption of vitamin A and haem iron rich foods. Vitamin A can be obtained from foods, either as preformed retinoids (with biological activity of retinol) in animal products or as pro-vitamin A Carotenoids, mainly $\beta$-carotene in plant products. Vitamin A and haem iron intake are usually expressed as retinol activity equivalent (RAE) and $\mathrm{mg} / 100 \mathrm{~g}$, respectively. The recently accepted conversion factor for pro-vitamin A such as $\beta$-carotene is 12 , for $\beta$-cryptoxanthin and $\alpha$-carotene is 24 , meaning that $12 \mu \mathrm{g} \beta$-carotene and $24 \mu \mathrm{g} \beta$-cryptoxanthin or $\alpha$-carotene, respectively, supposedly exert the activity of $1 \mu \mathrm{g}$ vitamin A $[19,20]$.

Percentages for consumption of vitamin A and haem iron rich food groups were estimated using the first day data. Of 640 participants, $39.7 \%$ consumed either plant or animal source of vitamin A and $13.7 \%$ consumed organ meat, flesh meat or fish source of haem iron. In other words, the risk of vitamin A deficiency was $60.3 \%$ and about $86 \%$ of the consumers did not obtain animal source of iron. The consumption of Animal Source Food (ASF) was 35.4\%.

The odds of consuming all types of vitamin A rich food groups in urban settings were 1.69 times higher than the odds of rural settings. Similarly, haem iron rich food groups were 9 times more likely to be consumed in urban settings than rural settings Table 10. As indicated in Table 11, the consumption of food groups rich in vitamin A and haem iron were significantly different across FVS and DDS $(P<0.05)$.

\section{Vitamin D rich foods and iodized salt consumptions}

Vitamin D rich food sources such as fish, organ meat and sun treated mushroom were consumed by $3.6 \%, 2.6 \%$ and $4.0 \%$ of participants, respectively. This indicated that more than $95 \%$ of the study participants were at risk of vitamin D deficiencies. Salt iodization is basically a safe and effective strategy for the prevention and control of iodine deficiency disorders. Salt consumption rate was very high, however, only $69.5 \%$ of subjects used iodized salt in their diet. This was far below the target of WHO iodine coverage (90\%) [21].

\section{Food taboos}

About $20 \%$ of participants avoided one or more food items from their diet. Bread (3.1\%), milk (2.8\%), fermented injera $(1.7 \%)$, raw beef $(1.7 \%)$ and tomatoes $(1.3 \%)$ were the major food items reported as food taboos. Table 12 shows the percentages of avoided food items from consumption.

\section{Nutritional status}

Anthropometric measurements showed that the median 
Table10: Indicators for the consumption of vitamin A and haem-iron rich food groups

\begin{tabular}{|c|c|c|c|c|c|c|c|c|c|}
\hline \multirow[b]{2}{*}{ Food groups } & & \multirow[b]{2}{*}{$\begin{array}{l}\text { Percent } \\
(n=640)\end{array}$} & \multicolumn{2}{|c|}{ Urban vs Rural } & \multirow[b]{2}{*}{$\begin{array}{c}\text { Pearson } \\
\text { Chi-Square }\end{array}$} & \multirow[b]{2}{*}{ P-value } & \multirow[b]{2}{*}{$\begin{array}{l}\text { Odds } \\
\text { Ratio }\end{array}$} & \multirow{2}{*}{$\begin{array}{c}95 \% \text { CI } \\
\text { LL }\end{array}$} & \multirow[b]{2}{*}{ UL } \\
\hline & & & Urban & Rural & & & & & \\
\hline \multirow{2}{*}{$\begin{array}{l}\text { Plant Vitamin A } \\
\text { (Pro-vitamin A) }\end{array}$} & Yes & 15.8 & 81 & 20 & 30.78 & $<0.0001^{*}$ & 4.03 & 2.39 & 6.71 \\
\hline & No & 84.2 & 270 & 269 & & & & & \\
\hline \multirow{2}{*}{ Animal Vitamin A (Pre-vitamin A) } & Yes & 29.5 & 109 & 80 & 0.78 & 0.377 & 1.18 & 0.83 & 1.65 \\
\hline & No & 70.5 & 242 & 209 & & & & & \\
\hline \multirow{2}{*}{ All Vitamin A } & Yes & 39.7 & 159 & 95 & 9.84 & $0.002^{*}$ & 1.69 & 1.21 & 2.31 \\
\hline & No & 60.3 & 192 & 194 & & & & & \\
\hline \multirow{2}{*}{ Animal Source Food } & Yes & 35.5 & 142 & 85 & 8.13 & $0.004^{*}$ & 1.63 & 1.16 & 2.25 \\
\hline & No & 64.5 & 209 & 204 & & & & & \\
\hline \multirow{2}{*}{ Haem Iron } & Yes & 13.7 & 79 & 9 & 49.86 & $<0.001^{*}$ & 9.04 & 3.61 & 18.2 \\
\hline & No & 86.2 & 272 & 280 & & & & & \\
\hline
\end{tabular}

*Statistically significant

Table 11: Vitamin A and haem iron rich food groups consumption across FVS and DDS

\begin{tabular}{|c|c|c|c|c|c|c|c|c|c|}
\hline \multirow[b]{2}{*}{ Scores } & \multirow[b]{2}{*}{ Categories } & \multicolumn{3}{|c|}{ Vitamin A consumption } & \multirow[b]{2}{*}{ P-value } & \multicolumn{3}{|c|}{ Haem iron consumption } & \multirow[b]{2}{*}{ P-value } \\
\hline & & $\begin{array}{c}\text { Yes } \\
n=251\end{array}$ & $\begin{array}{c}\text { No } \\
n=386\end{array}$ & $\begin{array}{c}\text { Chi-square } \\
\text { test }\end{array}$ & & $\begin{array}{c}\text { Yes } \\
n=88\end{array}$ & $\begin{array}{c}\text { No } \\
n=549\end{array}$ & $\begin{array}{c}\text { Chi-square } \\
\text { test }\end{array}$ & \\
\hline \multirow{4}{*}{$\begin{array}{l}\text { Food variety } \\
\text { score }\end{array}$} & $<10 \mathrm{FVS} /$ week & 0.8 & 1.3 & 13.15 & $0.004^{*}$ & 0.0 & 1.3 & 16.01 & $0.001^{*}$ \\
\hline & 10-19FVS/week & 75.3 & 85.5 & & & 68.2 & 83.6 & & \\
\hline & 20-24FVS/week & 23.5 & 13.2 & & & 31.8 & 14.9 & & \\
\hline & 25-30FVS/week & 0.4 & 0.0 & & & 0.0 & 0.2 & & \\
\hline \multirow{4}{*}{$\begin{array}{l}\text { Diet diversity } \\
\text { score }\end{array}$} & $<2.50$ DDS & 0.8 & 0 & 44.09 & $<0.001^{*}$ & 2.3 & 0 & 17.6 & $0.001^{*}$ \\
\hline & 2.50-3.50 DDS & 27.1 & 50.5 & & & 29.5 & 43.2 & & \\
\hline & 3.51-4.50 DDS & 64.9 & 47.9 & & & 63.6 & 53.2 & & \\
\hline & 4.51-5.50 DDS & 7.2 & 1.6 & & & 4.5 & 3.6 & & \\
\hline
\end{tabular}

body weight, height and BMI were 60 (range $=37$ to 89) $\mathrm{kg}, 1.65$ (range= 1.2 to 1.9 ) $\mathrm{m}$ and 22.05 (range $=13.49$ to 40.21 ) kg/ $\mathrm{m}^{2}$, respectively. Men and women had statistically significant difference in body weight and height measurements $(P<0.001)$ Our study revealed that $6.9 \%$ of subjects were underweight, $17.1 \%$ were overweight and $4.1 \%$ were obese. This implied that the proportion of malnutrition in the area was $28.1 \%$. Unlike body weight and height, men and women did not have a significant difference in BMI $(\mathrm{P}=0.164)$. But, there was a statistically significant difference in BMI across urban and rural settings $(\mathrm{P}=0.028)$ Table 6. The link between BMI and DDS was limited.

\section{Discussion}

\section{Dietary Patterns}

We identified about 130 food items. The major dietary patterns composed of cereals (teff, wheat and barley), vegetables (onion, green pepper, tomato and cabbage), legumes (peas, faba bean and lentils), oils (cooking oil) and spices (salt). All these food items are the ingredients of injera and thick stew made from flour of roasted legumes ('shiro wot'). The trend of taking hot beverages (coffee and tea) with sweets (sugar) was habituated by almost all people. In line with this finding, other studies also reported cereals, vegetables, legumes and oils as the main staples in Ethiopia [16, 22]. 
Relying on such dietary patterns implied that starchy staples and legumes are the predominant sources for energy and protein, respectively. Energy-dense foods, especially mixtures of sugars and fat, tend to be more palatable than foods of low energy density and high-water content [23]. Excessive intake of beverages and sweets containing added sugar could be a driving force behind obesity epidemic [24].

Less frequently, food groups containing dairy products (milk, butter, butter milk, yogurt and cheese), dark green vegetables (kale, spinach and lettuce), meat (beef, lamb, goat meat and chicken) and other pro-vitamin A rich fruits and vegetables (apricots, mango, carrots and pumpkins) were included in the dietary patterns. This was substantiated by the reports of Workicho, et al. and Amare, et al. in which they indicated that fruits and animal products were less frequently consumed in Ethiopia [16, 25]. Although the country has a very large livestock population, the availability of meat and other animal products for local human consumption is limited mainly due to economic reasons [26].

We also identified that the dietary patterns rarely entailed food groups containing fish (fresh fish) and organ meat (liver, kidney, heart and tripe). Despite abundant resources, fish consumption in Ethiopia is very limited. This is due to cultural factors and poor connections between production areas and markets. Fish is mostly consumed in large towns during periods of religious fasting [26]. There was also a limited access to organ meats. One of the reasons could be infection. Most of the time, livers from cattle and sheep are infected by internal parasites and as the result they are condemned from consumption.

Our results showed that dietary patterns were significantly different in urban and rural settings $(\mathrm{P}<0.05)$. The difference could be attributed to availability and accessibility of food groups. Urban people are very close to the market where much variety of foods could be available. Although all people could not have equal access to food varieties because of affordability, the case for rural people is even worse. Large numbers of rural people are living at subsistence level and far away from the market so that they have less access and economic power to purchase food varieties with high price.

\section{Meal frequency}

The median meal frequency per day was 3 with a range of 1 to 5. But, another study reported a range of 2 to 3 meal frequency per day [27]. This implied that the lowest consumption rate was 1 and the highest was 5 . The consumption rate significantly varied in urban and rural areas $(P<0.001)$. This could be justified by the difference in food groups availability and accessibility in urban and rural settings.

\section{Food varieties}

The median FVS was 16 per week but the range varied from 8 to 25 per week. Urban dwellers had a higher FVS as compared to rural dwellers. Based on the classification made by Savige, et al. $98 \%$ of people had FVS between poor and fair dietary adequacy
[12]. This result is prone to seasonal fluctuation. The scenario could be even worse during low production season in rural settings as the present study was conducted during harvesting season. FVS had a positive correlation with DDS ( $\mathrm{r}=0.502$, $P<0.0001)$ and BMI $(r=0.145, P<0.0001)$. But, it had a negative correlation with average meal frequency $(r=-0.102, P=0.01)$. This inverse association suggested a monotonous type of diet. Besides, Kant explained that consuming some food items more frequently means that other food items are being consumed less frequently [28].

Socio demographic characteristics such as educational status and family size were linked to FVS, but their impacts were different. Educational status had a positive influence on FVS. This implied that educated persons better understand the health benefit of consuming nutritious foods and spend much budget on food varieties. This was corroborated by other studies done in Ethiopia and in Tanzania [16, 29]. On contrary, family size had a negative influence on FVS. Increasing the members of family without increasing income could deter the access to food varieties. Income and supply of foods had great impacts on the dietary diversity of food consumed [30].

\section{Dietary diversity}

Dietary diversity is a qualitative measure of food consumption which evaluates the dietary quality of the individuals. Based on the guideline for individual DDS nine food groups were used for assessment and the median DDS was 3.43 (range $=1.14$ to 5.57). This was comparable with the mean DDS of 3.4 reported by Weldehaweria, et al. among lactating women in Tigray, Ethiopia [13]. In the same region, another study reported a median of 5 DDS, but 14 food groups were used for evaluation [22]. Hence, it is very difficult to compare the three results owing to the difference in study subjects and number of food groups used for counting. The implication of the median 3.43 DDS was that half of the people at least included 3 food groups in their diet. Mostly, starchy staples, vegetables and legumes were involved in the dietary patterns.

About $96 \%$ of people in the study areas had DDS below an average 4.5. A diet of at least 4 DDS was valid as nutritionally adequate, but below 4 DDS represented poor diversity [32, 33]. DDS was directly associated with BMI and average meal frequency. After controlling for age group, occupation, marital status and family size, the influence of educational status on DDS was significant. This was supported by Workicho, et al. and Mbwana, et al. [16, 29]. However, there were reports that showed the inverse relationship between education and DDS [25, 34, 35]. The explanation given was that although some people particularly women were educated; their employment rate was very low and have less income as the result they relied on poor nutrition.

\section{Vitamin A and iron indicators}

DDS can be used as a proxy indicator to assess the likelihood of achieving micronutrient requirements [16]. Micronutrients can be obtained from vegetables, fruits and Animal Source Foods 
(ASF). Failing to consume such kind of foods regularly may impair the immune systems and prone to infectious diseases. In the present study, consumption of vitamin A and haem iron rich food groups were assessed using the first day data of food consumption.

The results showed that $39.7 \%$ of people consumed either plant or animal source of vitamin A and $13.7 \%$ consumed organ meat, flesh or fish as source of haem iron. This implied that the remaining $60.3 \%$ was at risk of vitamin A deficiency and $86.3 \%$ was unable to get haem iron sources of foods. According to WHO definition, when food groups with high vitamin A content are consumed less than three times in a week by three fourth or more of vulnerable groups, there is a high risk of inadequate vitamin A status [36]. Given this definition, the result of the present study revealed that there were high risks for vitamin A and iron deficiencies in Central Ethiopia.

\begin{tabular}{|c|c|c|}
\hline Food group & Food item & Percen \\
\hline \multirow{4}{*}{ Vegetables } & Lettuce & 0.94 \\
\hline & Kale & 0.63 \\
\hline & Tomatoes & 1.26 \\
\hline & Beetroot & 0.47 \\
\hline Fruits & Avocado juice & 0.47 \\
\hline \multirow{4}{*}{ Cereals } & Bread & 3.14 \\
\hline & Porridge & 1.1 \\
\hline & Roasted wheat & 1.1 \\
\hline & Fermented injera & 1.73 \\
\hline \multirow{2}{*}{ Legumes and seeds } & Lentils & 0.47 \\
\hline & Linseeds & 0.47 \\
\hline \multirow{2}{*}{ Meat and Fish } & Raw beef & 1.73 \\
\hline & Goat meat & 0.47 \\
\hline Eggs & Fried egg & 0.47 \\
\hline \multirow{3}{*}{ Dairy products } & Milk & 2.83 \\
\hline & Yogurt & 0.63 \\
\hline & Cheese & 0.47 \\
\hline \multirow[t]{2}{*}{ Salts and sugar } & Salt & 0.94 \\
\hline & Sugars & 0.47 \\
\hline
\end{tabular}

We compared the consumption of vitamin A and haem iron rich food groups in urban and rural settings. The results showed that urban people were nearly 1.7 times and 9 times more likely to consume vitamin A and haem iron rich food groups than rural people, respectively. This, in turn, implied that the risks of vitamin A and iron deficiencies would be higher among rural communities than urban communities. This was corroborated by the report of Alaofe, et al. that vitamin A and iron deficiencies were high among rural communities particularly women and their children

in Northern Benin [37]. The consumption of vitamin A and haem iron rich food groups was significantly different across FVS and DDS $(P<0.05)$. This suggested that people who are consuming less variety of food items and diversity of food groups are less likely to incorporate food groups rich in vitamin A and haem iron in their diet.

\section{Vitamin D and iodine indicators}

Low intake of vitamin D was realized in the study areas. Very few people consumed fish (3.6\%), organ meat $(2.6 \%)$ and sun treated mushrooms (4\%). Although vitamin D rich foods are the second sources of vitamin D next to sunshine Keflie, et al. in their review paper reported the predictors of vitamin D deficiencies in tropical African countries. Taking the predictor factors into account, the risk of vitamin D deficiencies could be anticipated to be very high. Iodine consumption was also assessed, and the result revealed that $69.4 \%$ of people consumed iodized salt albeit high salt consumption rate. This implied that salt iodization coverage did not reach to at least three fourth of the communities. However, WHO suggested $90 \%$ coverage for the prevention and control of iodine deficiency disorders [21]. In other words, more than one fourth of the people are at risk of iodine deficiency disorders.

\section{Food taboos}

Food taboos were another concern. One fifth of people avoided one or more food items from their diet. The major reasons ascribed to food taboos were culture and health related problems. Some people usually avoided consumptions of raw foods such as tomatoes, vegetables, beef and milk due to their fear for infectious diseases; fermented injera and bread for stomach ache; and eggs and goat meat for cultural reasons.

\section{Nutritional status}

The results of BMI (median=22.05; range $=13.49-40.21 \mathrm{~kg} /$ $\mathrm{m}^{2}$ ) illustrated that $28.1 \%$ of people were malnourished. Of whom, $6.9 \%$ were underweight, $17.1 \%$ were overweight and $4.1 \%$ were obese. The proportion of overweight people was larger than the proportions of underweight and obese. This suggested that overweight has become a serious public health concern followed by underweight and obesity. There is a perception among many Ethiopian communities that overweight and being obese are indicators for wealth and health status. BMI had a statistically significant difference across urban and rural settings. The proportion of overweight and obesity were like the report of Amare, et al. in Gondar town of Ethiopia (21.3\%) [25]. But, the proportion of overweight alone was higher than those reported among adolescent girls in rural Southern Ethiopia (13.8\%) [27]. This suggested in general that malnutrition is the major public health problem in Ethiopia.

After the socio demographic characteristics were controlled, the link between BMI and DDS became insignificant. Savy, et al. described that the socio demographic and economic context could reduce the strength of the link between nutritional status and DDS [8]. This was explained that nutritional status was not 
only determined by the quality of food but also the quantity of the foods consumed.

In several studies done elsewhere, height less than $1.45 \mathrm{~m}$ was used as a cut-off point for determination of stunting in women [39-41]. Based on this threshold, the anthropometric results indicated that $4.6 \%$ of people were stunted, of whom three fourth were women. The proportion of stunted women was slightly comparable to the report of $2.2 \%$ from a study among lactating women in Tigray, Ethiopia [22]. Even though, the proportion of underweight women was higher than that of men, the nutritional status was not significantly different. This showed that both men and women were affected by malnutrition without any difference. And hence, the nutritional intervention measures should give emphasis on both genders.

\section{Limitations}

Although this study has the strength of dealing with dietary patterns, nutritional adequacy and nutritional quality, the dietary assessment instruments used to define the dietary patterns are based on self-report and may inflict some levels of report bias. This study does not contain data on seasonal variations of food consumption as it is a community based cross-sectional study.

\section{Conclusions}

In conclusion, the people of North Shewa, Central Ethiopia have cereal and legume based dietary patterns. Almost all of them have poor dietary adequacy and nutritional quality, and $28.1 \%$ are malnourished. Overweight and obesity are the upcoming nutritional problems besides under nutrition. Family size and educational status determine FVS. But, the later determines DDS Related with low frequency of consumptions, the risks for vitamin A, vitamin D, iron and iodine deficiencies are very high. All these nutritional problems underlined the implications of nutritional interventions. Therefore, by giving emphasis on the improvement of food and nutrition security, and considering the real situations of the area, the following recommendations are made:

1. Improving meal frequency, food varieties and diet diversities;

2. Creating awareness of the nutritional benefits of consumption of locally available food items including edible wild plants like stinging nettle (Urticasimensis);

3. Demonstrating the idea of balanced diet in the garden or kitchen garden;

4. Developing food based dietary guidelines;

5. Promoting nutrition sensitive agricultural practices; and

6. Promoting micronutrient enriched staple food.

\section{Declarations}

\section{Competing interests}

The authors declare that they have no competing interest related with this study.

\section{Funding}

There was no specific grant for this study.

\section{Authors contributions}

Design of the study: TSK, CL and HKB.

Data collection, analysis, interpretation and draft of manuscript: TSK

Critical review of the manuscript: AS, CL, DN and HKB. All the authors read and approved the manuscript.

\section{Ethical approval and consent to participate}

This study is a part of our project which has been ethically approved by Armauer Hansen Research Institute (AHRI) - All Africa Leprosy Rehabilitation Training (ALERT) Centre ethical approval committee in Ethiopia. It has also obtained permissions from zonal and district level health bureaus. The purposes and objectives of the study were explained to the study subjects. After informing about their right to withdraw from the study at any time, informed consent was obtained from each study subject

\section{Availability of data and material}

All data supporting the conclusions of this study are included in this article [and its additional file].

\section{Acknowledgment}

The authors are grateful to study participants, data collectors and the health bureau of North Shewa zone of Amhara Region. This study was financially supported by the Dr. Hermann Eiselen Ph.D. Grant from the Foundation fiat panis. The first author obtained a scholarship from Food Security Centre of University of Hohenheim, which is supported by the German Academic Exchange Service (DAAD) with funds of the Federal Ministry of Economic Cooperation and Development (BMZ) of Germany, and thus, we are indebted for this.

\section{References}

1. USDA. Scientific report of the 2015 dietary guidelines advisory committee. Advisory report to the secretary of health and human services and the secretary of agriculture.

2. Pinstrup-Andersen P. Food security: definition and measurement. Food Sec. 2009;1(1):5-7.

3. Sibhatu KT, Krishna VV, Qaim M. Production diversity and dietary diversity in smallholder farm households. Proc Natl Acad Sci U S A. 2015;112(34):10657-10662. doi: 10.1073/pnas.1510982112

4. Hassen IW, Mekdim D, Bart M, Kalle H. Diet transformation in Africa: The case of Ethiopia. ESSP Working Paper 87. Washington, D.C. and Addis Ababa, Ethiopia: International Food Policy Research Institute (IFPRI) and Ethiopian Development Research Institute (EDRI);2016.

5. Ayele Z, Peacock C. Improving access to and consumption of animal source foods in rural households: the experiences of a women-focused goat development program in the highlands of Ethiopia. J Nutr. 2003;133(11 Suppl 2):3981S-3986S. 
6. Speedy AW. Global Production and Consumption of Animal Source Foods. J Nutr.2003;133(11 Suppl 2):4048S-4053S.

7. Melaku YA, Temesgen AM, Deribew A, Tessema GA, Deribe K, Sahle BW, et al. The impact of dietary risk factors on the burden of noncommunicable diseases in Ethiopia: findings from the Global Burden of Disease study 2013. International Journal of Behavioural Nutrition and Physical Activity. 2016;13:122. doi: 10.1186/s12966-016-0447-x

8. Savy M, Martin-Pre'vel Y, Sawadogo P, Kameli Y, Delpeuch F. Use of variety/diversity scores for diet quality measurement: relation with nutritional status of women in a rural area in Burkina Faso. Eur J Clin Nutr. 2005;59(5):703-716. doi:10.1038/sj.ejcn.1602135

9. Mirmiran P, Azadbakht L, Esmaillzadeh A, Azizi F. Dietary diversity score in adolescents a good indicator of the nutritional adequacy of diets: Tehran lipid and glucose study. Asia Pac J Clin Nutr. 2004;13(1):56-60.

10. Clausen T, Charlton KE, Gobotswang KSM, Holmboe-Ottesen G. Predictors of food variety and dietary diversity among older persons in Botswana. Nutrition. 2005;21(1):86-95. Doi: 10.1016/j. nut.2004.09.012

11. Ruel MT. Operationalizing dietary diversity: A review of measurement issues and research priorities. J Nutr. 2003;133:S3911-S3926.

12. Savige G, Hsu-Hage B, Wahlqvist ML. Food variety as nutritional therapy. Current therapeutics. 1997.

13. FAO. Food and Agriculture Organization. Guidelines for measuring household and individual dietary diversity. Version 2. Rome, Italy; 2007.

14. Foote JA, Murphy SP, Wilkens LR, Basiotis PP, Carlson A. Dietary variety increases the probability of nutrient adequacy among adults. J Nutr. 2004;134(7):1779-1785.

15. Arimond M, Wiesmann D, Becquey E, Carriquiry A, Daniels M, Deitchler $\mathrm{M}$, et al. Simple food group diversity indicators predict micronutrient adequacy of women's diets in 5 diverse, resource -poor settings. J Nutr. 2010;140(11):2059S-2069S

16. Workicho A, Belachew T, Feyissa GT, Wondafrash B, Lachat C, Verstraeten R, et al. Household dietary diversity and Animal Source Food consumption in Ethiopia: evidence from the 2011 Welfare Monitoring Survey. BMC Public Health. 2016;16:1192.

17. CSA. Ethiopia Population and Housing Census. Population and housing census 2007 Report, Amhara, Part I: population size and characteristics.

18. WHO. Report of a World Health Organization Expert Committee on Physical status: the use and interpretation of anthropometry. Geneva. WHO;1995.

19. Institute of Medicine. Dietary Reference Intakes for Vitamin A, Vitamin $\mathrm{K}$, Arsenic, Boron, Chromium, Copper, Iodine, Iron, Manganese, Molybdenum, Nickel, Silicon, Vanadium, and Zinc. A Report of the Panel on Micronutrients, Subcommittees on Upper Reference Levels of Nutrients and of Interpretation and Uses of Dietary Reference Intakes, and the Standing Committee on the Scientific Evaluation of Dietary Reference Intakes Food and Nutrition Board. IOM; 2001. National
Academy Press, Washington, DC, 91-93.

20. Weber D, Grune T. The contribution of b-carotene to vitamin A supply of humans. Review. Mol Nutr Food Res. 2012;56(2):251-258. doi: 10.1002/mnfr.201100230

21. WHO. Assessment of Iodine Deficiency Disorders and Monitoring Their Elimination: A Guide for Programme Managers, Geneva. WHO/ UNICEF/ICCIDD; 2007.

22. Haileslassie K, Mulugeta A, Girma M. Feeding practices, nutritional status and associated factors of lactating women in SamreWoreda, South Eastern Zone of Tigray, Ethiopia. Nutr J. 2013;12:28. doi: 10.1186/1475-2891-12-28

23. Drewnowski A. The role of energy density. Lipids. 2003;38(2):109 -115. doi:10.1007/s11745-003-1039-3

24. Drewnowski A, Mennella JA, Johnson SL, Bellisle F. Sweetness and Food Preference. J Nutr. 2012;142(6):1142S-1148S. doi: 10.3945/ jn.111.149575

25. Amare B, Moges B, Moges F, Fantahun B, Admassu M, Mulu A, et al. Nutritional status and dietary intake of urban residents in Gondar, Northwest Ethiopia. BMC Public Health. 2012;12:752.

26. Food and Agriculture Organization. Ethiopia Nutrition Profile Nutrition and Consumer Protection Division. FAO;2008.

27. Roba AC, Gabriel-Micheal K, Zello GA, Jaffe J, Whiting SJ, Henry CJ. A Low Pulse Food Intake May Contribute to the Poor Nutritional Status and Low Dietary Intakes of Adolescent Girls in Rural Southern Ethiopia. Ecol Food and Nutr. 2015;54(3):240-254. doi: 10.1080/03670244.2014.974593

28. Kant AK. Dietary Patterns and Health Outcomes: Review. J Am Diet Assoc. 2004;104(4):615-635.

29. Mbwana H, Kinabo J, Lambert C, Biesalski HK. Determinants of household dietary practices in rural Tanzania: Implications for nutrition interventions. Cogent Food and Agriculture. 2016;2(1):1224046.

30. Food and Agriculture Organization. Agriculture, food and nutrition for Africa. A resource book for teachers of Agriculture. Rome: Food and Nutrition Division. FAO; 1997.

31. Weldehaweria NB, Misgina KH, Weldu MG, Gebregiorgis YS, Gebrezgi $\mathrm{BH}$, Zewdie SW, et al. Dietary diversity and related factors among lactating women visiting public health facilities in Aksum town, Tigray, Northern Ethiopia. BMC Nutrition. 2016;2:38. doi: 10.1186/s40795016-0077-3

32. Habte TY, Krawinkel M. Dietary Diversity Score: A Measure of Nutritional Adequacy or an Indicator of Healthy Diet. Journal of Nutrition and Health sciences. 2016;3(3). doi: 10.15744/23939060.3 .303

33. Drewnowski A, Henderson S, A, Driscoll A, Rolls BJ. The dietary Variety Score: assessing diet quality in healthy young and older adults. J Am Diet Assoc. 1997;97(3):266-271.

34. Sirotin N, Hoover D, Shi Q Adedimeji A, Mutimura E, Cohen M, et al. Structural determinants of food insufficiency, low dietary diversity and BMI: A cross-sectional study of HIV-infected and HIV-negative Rwandan women. 2012. doi:10.1079/PHN2005912 
35. Bhandari S, Sayami JT, Thapa P, Sayami M, Kandel BP, Banjara MR. Dietary intake patterns and nutritional status of women of reproductive age in Nepal: findings from a health survey. Arch Public Health. 2016;74:2. doi:10.1186/s13690-016-0114-3

36. WHO. Indicators for Assessing Vitamin A Deficiency and their application in monitoring and evaluating intervention programs. WHO, Geneva, Switzerland.WHO;1996.

37. Alaofè H, Burney J, Naylor R, Taren D. Prevalence of anaemia, deficiencies of iron and vitamin $\mathrm{A}$ and their determinants in rural women and young children: a cross-sectional study in Kalalé district of northern Benin. Public Health Nutr. 2017;20(7): 1203-1213.

38. Keflie TS, Nölle N, Lambert C, Nohr D, Biesalski HK. Vitamin D deficiencies among tuberculosis patients in Africa: A systematic review. Nutrition. 2015;31(10):1204-1212.
39. Girma W,Genebo T. Determinants of Nutritional Status of Women and Children in Ethiopia. ORC Macro Calverton, Maryland, USA. 2002.

40. ACC/SCN. Administration Committee on Coordination-Sub-Committee on Nutrition. Second report on the world nutrition situation, Vol. 1 and 2, Global and regional results. New York. 1992.

41. Krasovec K, Anderson MA. Maternal nutrition and pregnancy outcomes: Anthropometric assessment. Scientific Publication No. 529. Washington D.C. Pan American Health Organization. 1991.

42. USDA. United States Department of Agriculture. Agricultural Service National Nutrient Database for Standard reference release 28 slightly revised May 2016. Software developed by the National Agricultural Library v.3.7.1.USDA; 2017 